Revista Ingeniería y Región. 2016;16(2): 35-48

http://dx.doi.org/10.25054/22161325.1297

\title{
Efectos en la resistencia del hormigón simple elaborados con agua residual tratada proveniente de la PTAR Cañaveralejo
}

\section{Effects concrete strength simple for use of residual water mix treated from WWTP Cañaveralejo}

\author{
Andrés Saúl Calderón Linares ${ }^{1}$, Maria Jeimy Burbano Cerón²
}

\begin{abstract}
Resumen
En esta investigación se analizó el comportamiento de la resistencia de mezclas de hormigón preparados con agua residual tratada. Se seleccionó la "PTAR Cañaveralejo"; que trata las aguas residuales domesticas provenientes de la ciudad de Cali. Del efluente de la planta se analiza las variaciones de las concentraciones de los contaminantes en el agua, estas a su vez se compararon con los límites permisibles establecidos por las normas ASTM C-94 y NTC 3459; información correspondiente al año 2015 e inicios del 2016. Todas las concentraciones están dentro de los límites excepto la Materia Orgánica expresada como $\mathrm{DQO}$ y $\mathrm{DBO}_{5}$. Con muestras de agua residual tratada se procedió, a la elaboración de mezcla fresca de hormigón. El encofrado, curado y pruebas de resistencia de estos cilindros de hormigón, están bajo los lineamientos de la Norma INVIAS 2012. Los cilindros elaborados con agua residual fueron comparados con cilindros de agua potable proveniente del acueducto "El Tablazo" de la ciudad de Popayán. La resistencia en cilindros realizados con agua residual tratada diluida al 50\% con agua potable, alcanzó un $100 \%$ de resistencia a los 28 días, respecto a cilindros con agua potable (muestra patrón). Cilindros con un $100 \%$ de agua residual tratada obtuvieron resistencias del $90 \%$ a los 28 días respecto a cilindros con agua potable. El uso de agua residual tratada diluida no afecta la resistencia del hormigón demostrando un potencial uso a futuro, mayor investigación en el uso total de la misma debido a que se encuentra en el valor límite de aceptación según norma INVIAS 630 de 2013. Mezclas similares se hicieron con agua residual en reposo durante 8 días, obteniéndose resultados en resistencia similares a sus homólogos sin reposar. El agua reposada durante 8 días no afecta significativamente la resistencia del hormigón respecto a la no reposada.
\end{abstract}

Palabras clave: hormigón; agua residual tratada; resistencia.

\begin{abstract}
The resistance of concrete mixture's behavior prepared with treated wastewater was analyzed in this research. The WWTP "Cañaveralejo" was selected; which domestic wastewater are treat from the city of Cali. From the plant's water flow the research analyzes the variations of the pollutants in the water concentrations. These waters, at the same time, were compared with the permissible limits established by the ASTM C-94 and NTC 3459 standards; this information is
\end{abstract}

1 Ingeniero Ambiental. Universidad del Cauca (Colombia). Calle 5 No.4-70, Popayán (Colombia). andressaulcalderon@ gmail.com

2 Ingeniera Ambiental. Universidad del Cauca (Colombia). Calle 5 No.4-70, Popayán (Colombia). majebuce@gmail.com 
corresponding to the year 2015 and the beginning of 2016. All concentrations are within the limits except Organic Matter expressed as COD and BOD5. A fresh concrete mixture was produced with treated wastewater samples. The formwork, curing and resistance tests of these concrete cylinders follow the guidelines of the INVIAS 2012 Standard. The cylinders made with wastewater were compared with cylinders of drinking water from "El Tablazo" aqueduct of the city of Popayan. The resistance in cylinders made with treated wastewater diluted to $50 \%$ with potable water and reached $100 \%$ resistance at 28 days, compared to cylinders with potable water (standard sample). Cylinders with $100 \%$ treated wastewater obtained resistance of $90 \%$ at 28 days in relation to cylinders with potable water. Diluted treated wastewater use does not affect the concrete's resistance demonstrating potential future use, further investigation into the total use of the same because it is in the limit value of acceptance according to INVIAS 630 of 2013. Similar mixtures are made with residual water at rest for 8 days, resistance results were obtained similar to their non-standing counterparts. The water rested for 8 days does not significantly affect the strength of the concrete compared to the non-rested concrete.

Keywords: concrete; treated wastewater; resistance.

\section{Introducción}

La industria de la construcción es un importante consumidor de grandes cantidades de materiales naturales como el agua (Naik, 2007).

El 40\% de las materias primas en el mundo, que equivalen a 3000 millones de toneladas por año, son destinadas para la construcción, lo mismo sucede con el $17 \%$ del agua potable (Acevedo, et al, 2012). (Vásquez, et al., 2001) (Cámara Colombiana de la construcción CAMACOL., 2014).

El agua utilizada en la elaboración del hormigón y mortero por lo general debe ser apta para el consumo humano (agua potable), libre de sustancias como aceites, ácidos, sustancias alcalinas y materias orgánicas. (Reglamento Colombiano de Construcción Sismo- resistente, 2010).

Sin embargo, las fuentes hídricas enfrentan diferentes problemáticas como la contaminación y la escasez. Este fenómeno se presenta debido al manejo irresponsable de los residuos domésticos e industriales y al abuso de este recurso; por otra parte, la carencia también es debida a los bajos niveles de precipitación o por insuficientes cuerpos de agua. Algunas zonas geográficas presentan escasez económica ya que, a pesar de la existencia de agua, no se cuenta con la infraestructura necesaria para aprovecharla (Oviedo, 2011).

En Colombia, la industria de la construcción presenta gran tendencia de crecimiento debido a la edificación de viviendas de interés social, prioritario y privado. (Cámara Colombiana de la construcción CAMACOL., 2014.). Esto representa de igual manera uso de agua potable para mezclas del hormigón requerido.
Al presentarse situaciones en donde el uso de agua potable es limitado, evidencia que la industria de la construcción, tendría que entrar en una competencia con la demanda de agua para el consumo humano, la agricultura, otras industrias, y demás. Dando origen a una problemática que debe ser analizada y buscar posibles soluciones. Se han originado diferentes alternativas, como es el uso de agua lluvia para mezcla de concreto (Medina, 2013), sin embargo esta alternativa no es una solución definitiva.

El uso de agua residual tratada para la industria de la construcción se convierte en una posible solución definitiva a esta problemática(Diéguez, 2011), la reutilización de este tipo de aguas es una oportunidad de grandes empresas consumidoras de agua en demostrar un real compromiso con el medio ambiente.

El reúso de este tipo de aguas es una realidad, como es el caso de México, el trabajo denominado: "Comparación entre concretos elaborados con agua potable y concretos fabricados con agua residual tratada en Azcapotzalco"; se utiliza agua de diferentes PTAR, para evaluar la posibilidad de sustituir el agua potable en la elaboración de concreto, la mayoría de las muestras no presentan efectos negativos en la resistencia a la compresión, por el contrario se obtuvieron incrementos entre el 10\% y 40\% a los 28 días (Vásquez, et al, 2001).

La investigación "Uso de aguas residuales tratadas para la mezcla de concreto en Kuwait" pone a prueba la resistencia de cubos de hormigón elaborados con agua potable (A.P.), agua residual con tratamiento primario (A.R.T.P.), agua residual con tratamiento secundario (A.R.T.S.) y agua residual con tratamiento terciario (A.R.T.T.). El aumento del 
deterioro del agua de mezcla afecta directamente la resistencia del hormigón producido. (Al-Ghusain \&Terro, 2003).

La Investigación en Nigeria "Efectos de agua residual de fábrica de azúcar como la mezcla de agua en las propiedades de hormigón de baja resistencia"; utilizan proporciones de1 $75 \%$ y $100 \%$ de agua residual y un $100 \%$ de agua potable. Se encontró un aumento en el tiempo de fraguado inicial en mezclas con aguas residuales. La duración de curado se extendió a 90 días, los cubos de hormigón producidos en porcentajes con agua residual superaron la resistencia de muestra patrón. (Cubos con agua de mezcla de agua potable) (Gadzama, et al., 2015).

Este documento presenta el proceso de recolección, tratamiento y conversión de información correspondiente de los parámetros medidos en la PTAR Cañaveralejo y PTAP El Tablazo, para su consecuente comparación con normas que rigen en el ámbito de la construcción.

El diseño, elaboración y comparación entre muestras cilíndricas con agua residual y potable en su totalidad y en proporciones. Los resultados y análisis de estos procedimientos para concluir en un viable reúso por parte de empresas productoras de premezclados de hormigón y afines, así mismo, demostrar el gran potencial económico y ambiental de las PTAR.

\section{Metodología}

El lugar de recolección es la Planta de Tratamiento de Aguas Residuales (PTAR) Cañaveralejo, ubicada dentro del perímetro urbano de la ciudad Santiago de Cali, en el Departamento del Valle del Cauca. Este complejo de instalaciones se encuentra dispuesto con límites sobre la margen izquierda del río Cauca. El agua residual tratada es de tipo doméstico proveniente de las residencias de la ciudad.

\subsection{Recolección de información de parámetros medidos por PTAR}

\subsubsection{Evaluación de parámetros medidos por PTAR para el año 2015 y comienzos del 2016 res- pecto a norma ASTM C-94}

Para determinar la viabilidad de usar el efluente tratado de la PTAR Cañaveralejo, se solicitó información de los resultados de los parámetros que se midieron en el año 2015 hasta abril del 2016. Esta información fue tratada mediante el programa Excel obteniéndose los valores máximo, mínimo, promedio, varianza, desviación y coeficientes de variación de cada mes.

Estos valores a su vez se evaluaron respecto a la norma ASTM C-94 (American Society for Testing and Materials, ASTM C-94, 1994), pero antes se requirió de una estimación o de un proceso de conversión a sustancias específicas que la norma exige. Tal es el caso de: Cloruros a Cloruro de Sodio, Cloruro de Magnesio y Cloruro de Calcio; Alcalinidad a Bicarbonatos; Sulfatos a Sulfato de Sodio y Sulfato de Magnesio; hierro total a Cloruro de Hierro y Sulfato de Hierro.

Por otro lado, se analizó la presencia de Carbonatos a través de los valores de $\mathrm{pH}$ y Alcalinidad, por último la presencia de Hidróxidos y Ácidos fuertes con el pH.

Algunos parámetros se evaluaron directamente con la norma como es el caso de: Aceite mineral como Grasas y Aceites, $\mathrm{pH}$, Materia Orgánica como DQO y $\mathrm{DBO}_{5}$; y Partículas en suspensión como SST.

Finalmente los valores máximos, mínimos y promedio de las sustancias: Materia Orgánica (DQO y $\mathrm{DBO}_{5}$ ); Partículas en suspensión (SST); pH; Bicarbonato (Alcalinidad); Aceite Mineral (Grasas y Aceites); Sales de Hierro $\left(\mathrm{FeSO}_{4}\right.$ y $\mathrm{FeCl}_{3}$ ); Cloruros $\left(\mathrm{NaCl}, \mathrm{MgCl}_{2}\right.$ y $\mathrm{CaCl}_{2}$ ); se tabularon y graficaron junto con los valores máximos permisibles de la norma.

\subsection{Recolección de agua residual tratada}

La recolección del agua residual tratada fue de la cámara final y realizada por el personal de la PTAR, una vez tomada cada muestra se almacenó en un cuarto frio a $4^{\circ} \mathrm{C}$ en el laboratorio de aguas dentro de la PTAR, se recolectaron $95 \mathrm{~L}$ para la mezcla compuesta.

Finalmente se procedió a envasar muestras en 4 galones plásticos de aproximadamente $20 \mathrm{~L}$ cada uno, también en 5 recipientes de vidrio ámbar de capacidad de $1 \mathrm{~L}$, dos de las muestras recolectadas en los recipientes de vidrio quedaron para los análisis que la planta realiza comúnmente.

Adicionalmente se dejó una muestra en un recipiente recolector de capacidad de $2 \mathrm{~L}$ el cual se dejó al aire libre durante 8 días dentro del laboratorio de aguas de la Planta, después de este tiempo se realizaron las respectivas pruebas de laboratorio en la PTAR. 
En los laboratorios de la facultad de ingeniería Civil de la Universidad del Cauca se efectuaron análisis de algas, detección de azucares y sulfitos.

\subsubsection{Parámetros medidos y entregados por la PTAR Cañaveralejo}

La PTAR Cañaveralejo realizo pruebas físico-químicas de los siguientes parámetros: $\mathrm{pH}, \mathrm{DBO}_{5}, \mathrm{DQO}$, S.T., S.S.T., S.S.V., S.S., Conductividad, Alcalinidad Total, Acidez, Cloruros, Detergentes Anionicos, Fenoles Totales, Cianuros Totales, Grasas y Aceites, Nitrógeno Total, Fosforo Total, Plomo Total, Cromo Total, Zinc Total, Hierro Total, Mercurio Total. Para las muestras de agua empleadas para la elaboración del hormigón se solicitó adicionar la determinación del contenido de sulfatos.

Respecto a la muestra de agua residual tratada y reposada (A.R.T.R) durante 8 días, los parámetros medidos fueron: $\mathrm{pH}, \mathrm{DQO}$, S.S.T., Sulfatos, Plomo Total, Cromo Total, Zinc Total, Hierro Total y Mercurio Total.

Todos los parámetros fueron medidos de acuerdo al Standard Methods for the Examination of Water and Wastewater, Edición 22; excepto los parámetros de DQO y Mercurio total.

\subsubsection{Parámetros medidos y entregados por la PTAP El Tablazo}

Para la elaboración de mezclas patrón se empleó agua potable, proveniente de la planta de tratamiento de agua potable (PTAP) El Tablazo, que abastece el área del parque industrial. Este servicio es prestado por el Acueducto y Alcantarillado de Popayán.

Se solicitó la información de los parámetros físico-químicos del agua potable del día que se realizaron las mezclas de hormigón.

Los parámetros medidos por la PTAP fueron: Turbiedad, Color, pH, Alcalinidad Total, Dureza Total, Cloruros, Sulfatos, Hierro, Aluminio, Nitritos, Nitratos, Olor, Conductividad y Temperatura. No se especificó el método de medición usado para cada parámetro.

\subsection{Ensayos adicionales para agua residual tratada}

En el laboratorio de Biología de la Facultad de Ciencias Naturales Exactas y de la Educación y en el laboratorio de Ingeniería Ambiental de la Facultad de ingeniería Civil de la Universidad del Cauca, se realizaron los siguientes ensayos: Prueba de Molisch para la detección de Azúcares, Detección de pigmentos para presencia de Algas y Prueba Yodimétrica para valorar sulfitos.

\subsection{Evaluación de parámetros respecto a las normas ASTM C-94 y NTC 3459}

Los resultados de los parámetros medidos por la PTAR y PTAP de las muestras de agua para mezcla de hormigón, requirieron de estimación o conversión a sustancias específicas de las normas ASTM C-94 y adicionalmente a NTC 3459 (Norma Técnica Colombiana 3459,2001 ), ya que esta norma presenta los requisitos acogidos por Colombia, respecto al agua de mezcla para hormigón.

\subsection{Lugar de elaboración de ensayos a materiales y mezclas de hormigón}

Las mezclas y ensayos se llevaron a cabo en Premezclados Del Cauca, Predelca S. A. S., es una fábrica de concretos premezclados para la construcción de pavimentos, rellenos estabilizados, morteros y cualquier tipo de obra civil, garantizando el cumplimiento de las especificaciones técnicas de sus productos dosificados en planta computarizada.

\subsection{Ensayos de laboratorio a los agregados}

Los agregados (arena y grava) fueron aportados por Predelca, este material es comprado a la empresa "CONEXPE", que lo extrae de canteras aledañas a la ciudad de Popayán-Cauca.

\subsubsection{Ensayo granulométrico para arena}

\section{Equipos e instrumentos}

Juego de tamices de tamaño: 3/8", No. 4, No. 8, No. 16, No. 30, No. 50, No. 100 y No. 200; balanza electrónica, cepillo, estufa, contenedores de aluminio con capacidades: $10 \mathrm{Kg}, 20 \mathrm{Kg}$ y $50 \mathrm{Kg}$

Procedimiento: Se realizó cuarteo a una muestra de $10 \mathrm{Kg}$ de arena hasta reducir a $1 \mathrm{Kg}$, esta muestra se vertió a un contenedor y se procedió a lavar cuidadosamente este material con agua de grifo, se puso a secar en estufa hasta secado total, se repitió el lavado y secado de la muestra hasta que el agua de lavado estuvo lo más transparente posible.

Después del último secado, se reposó y pesó nuevamente la muestra este valor se anotó para posteriores 
cálculos. Inmediatamente se procedió a tamizar la muestra empezando con el tamiz de $3 / 8$ ", el material retenido en el tamiz se trasvasó a un recipiente el cual se pesó en una balanza electrónica, se anotó el valor de este peso.

El material pasante del tamiz 3/8" se trasvasó al tamiz No. 4 de nuevo se pesó y anotó el valor de material retenido, este proceso se realizó sucesivamente hasta el último tamiz, el No. 200.

\subsubsection{Ensayo granulométrico para grava}

\section{Equipos e instrumentos}

Se utilizan los mismos equipos e instrumentos del ensayo granulométrico de la arena, pero con juego de tamices de tamaño: 1ํㅡ", 1", 3/4", 1/2", 3/8", No. 4, No. 8, No. 16, No. 30, No. 50, No. 100 y No. 200

Procedimiento: Se realizó cuarteo a una muestra de $50 \mathrm{Kg}$ de grava hasta reducir a $5 \mathrm{Kg}$, en adelante se realiza el mismo proceso que se explicó anteriormente en el ensayo granulométrico de la arena.

\subsubsection{Curva granulométrica}

Los resultados de cada ensayo se tabularon y se determina el porcentaje de material pasante respecto al peso del material total que fue lavado y secado repetidas veces. Con la información del porcentaje de material retenido se obtiene el porcentaje acumulado de material retenido y el porcentaje de material pasante.

\subsection{Cemento y aditivo para elaboración de mezcla de hormigón}

Para las mezclas de hormigón se usó cemento Portland tipo1 de marca CEMEX, este cemento es muy común y usado en construcciones simples, sus características deben cumplir con la normatividad respectiva (Norma Técnica Colombiana 30, 2001). El aditivo de gran poder retardante y plastificante TM-20 (Zika., 2012), se usó en todas las mezclas de hormigón.

\subsection{Diseño de mezcla de hormigón}

El diseño de mezcla es aportado por la empresa "Predelca" (premezclados del Cauca), este diseño constó de dos fases, la determinación de la humedad en los agregados y la cantidad de materiales. Estas fases se detallan a continuación.

\subsubsection{Determinación de la humedad en agregados}

Se tomó una muestra de $1000 \mathrm{~g}$ de grava, se constató este peso con una balanza electrónica, se secó en estufa hasta secado completo aparente, se dejó en reposo hasta enfriar y se procedió a pesar nuevamente. Este proceso se repitió para la arena pero con una muestra de $500 \mathrm{~g}$.

\subsubsection{Cantidad de materiales usados en mezcla de hormigón}

Con la información obtenida de humedad en los agregados, estos valores se introdujeron en un programa para diseño de mezcla con el cual cuenta Predelca, el programa arroja la cantidad en peso de los materiales que se usó para realizar las respectivas mezclas.

\subsection{Preparación de la mezcla y cilindros de hormigón}

Se pesaron todos los materiales hasta llegar a los valores indicados del diseño, para este proceso se usa una maquina mezcladora. Siguiendo el siguiente orden; la arena con la grava y se encendió la máquina, el tiempo de mezcla es de un minuto; se agrega el cemento y nuevamente se encendió la maquina durante un minuto aproximadamente, este proceso en su orden es para homogenizar los materiales en la mezcla.

El agua previamente pesada, se agregó gradualmente a la mezcla homogenizada dentro de la mezcladora; la adición de agua se realizó con la máquina en funcionamiento durante 3 minutos. Al finalizar la adición de agua, con la mezcladora en funcionamiento se agregó el aditivo líquido TM-20 lentamente en toda la mezcla.

\subsubsection{Prueba de asentamiento}

Para esto se empleó el Cono de Abrams, una varilla metálica o "punzón”, palustre y una cinta métrica.

Se llenó el cono de Abrams con la mezcla, en tres capas, al final de cada capa de llenado se "punzó" 25 veces con la varilla en forma circular alrededor de todo el cono en forma consistente, en las dos últimas etapas, se tuvo cuidado de que la varilla no penetrara en las capas anteriores.

Una vez terminado este proceso, se retiró cuidadosamente el cono, el cono se giró y sirvió como punto base de medición de la altura de mezcla. La altura 
recomendada por Predelca fue de $15 \mathrm{~cm}$, cuando se midió esta la altura obtenida fue inferior a $\operatorname{los} 15 \mathrm{~cm}$, de esta manera se corrigió adicionando más agua y se mezcló nuevamente, hasta que se llegó a este valor. Este proceso cumple con las especificaciones técnicas de la norma INVIAS sección 400 (Instituto Nacional de Vías, INVIAS).

\subsubsection{Encofrado o llenado de moldes cilíndricos}

Con los cilindros previamente limpios y ligeramente lubricados con aceite combustible para motores (ACPM), se procedió a llenar en tres capas y con cuidado de no punzar la capa anterior, al final de cada capa se dio golpes suaves alrededor del molde con un martillo de goma para que la mezcla se compactara y rellenaran posibles espacios de aire. Se enrasó con la varilla y se emparejó con el palustre.

Se rotularon con las fechas de elaboración y de rotura, hora de mezcla, tipo de mezcla y temperatura medioambiental; se dejaron en un espacio nivelado y protegido de la lluvia e intervenciones que puedan deformar la mezcla.

Pasadas 24 horas, se desencofraron y se dejaron en un tanque de inmersión (curado húmedo) y almacenamiento hasta la prueba de resistencia. Este proceso cumple con las especificaciones técnicas de la norma INVIAS sección 400 (Instituto nacional de vías, INVIAS).

\subsection{Diseño de cilindros de hormigón con agua residual de reposo}

Parte de la muestra de agua residual tratada se dejó en reposo durante 8 días (A.R.T.R.), en un galón plástico al aire libre dentro del laboratorio de Predelca, pasado este periodo de tiempo se utilizó esta agua para elaborar los tipos de mezcla. No se realizó mezcla patrón, se usó como referencia la mezcla de agua potable anteriormente realizada.

\subsection{Prueba de resistencia a la compresión}

La prueba consiste en manipular la máquina de prueba de resistencia (Modelo PC-160; marca PINZUAR, maquina Colombiana creada en el 2004), esta máquina aplica una carga axial de compresión a los cilindros moldeados con una velocidad gradual y continua, hasta que se presenta la falla o rompimiento.

La resistencia a la compresión se determinó dividiendo la máxima carga aplicada durante el ensayo entre la sección transversal del cilindro (área circular del cilindro).

Las pruebas de compresión se realizaron a los 7, 14 y 28 días para las mezclas generales, a cada día le correspondió respectivamente dos cilindros de muestra por cada tipo y proporción de agua de mezcla realizada.

Para las mezclas de agua residual tratada en reposo de 8 días, se realizaron pruebas de resistencia a los 7, 28 y 56 días. Alos 56 días le correspondió un solo cilindro respectivamente por cada tipo de agua de mezcla. Se midieron los perímetros de todos los cilindros, ya que este puede variar un poco e influir en cálculos posteriores.

En primera instancia se calculó el área de la cara circular de los cilindros donde se ejerce la fuerza por parte de la máquina, se usan ecuaciones básicas de perímetro y área de una circunferencia para obtener finalmente:

$$
\text { Area circunf }\left(\mathrm{cm}^{2}\right)=\frac{(\text { Perímetro circunf }(\mathrm{cm}))^{2}}{4 * \pi}
$$

En la ecuación (1) la fuerza medida por la maquina está en unidades de KN (Kilonewtons), los cuales deben ser convertidos a Kf(Kilogramos Fuerza) con la siguiente relación.

$$
1 K N=101,97 K f
$$

La presión ejercida se expresa entonces:

$$
\text { Presión }\left(\frac{K f}{\mathrm{~cm}^{2}}\right)=\frac{\text { Presión máquina }(\mathrm{Kf})}{\text { Area circunf }\left(\mathrm{cm}^{2}\right)}
$$

La presión ejercida en unidades de $\mathrm{Kf} / \mathrm{cm}^{2}$ puede ser expresada en P.S.I. con la siguiente relación:

$$
1 \mathrm{Kf} / \mathrm{cm} 2=14,22 \text { P.S.I. }
$$

\subsubsection{Tipos de fracturas}

La forma en que se rompen o fracturan los cilindros indica si la fuerza ejercida sobre este fue distribuida uniformemente, indica un buen procedimiento tanto en la elaboración de la mezcla y composición de buenos materiales. El tipo de factura que indica lo anterior es aquel que se produce en un ángulo de $45^{\circ}$ desde el extremo superior o inferior del cilindro (Rivera, 2000) (Figura 1).

\subsection{Análisis estadístico}

La unidad experimental de esta investigación fueron los cilindros elaborados con cada tipo de agua, las va- 


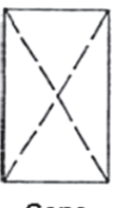

Cono
(a)

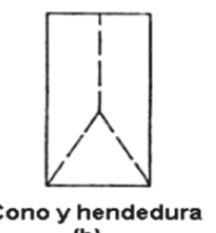

(b)

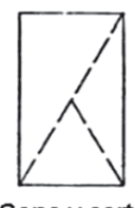

(c)

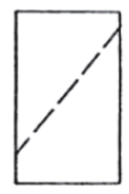

(d)

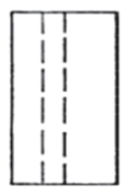

Columnar (e)
Figura 1. Tipos de fracturas.

riables de respuesta son los valores de resistencia a la compresión, el factor de tratamiento es la proporción $\mathrm{y}$ tipo de agua usados.

Los análisis estadísticos fueron realizados mediante el software estadístico SPSS versión 21 y Microsoft office Excel 2013. A los porcentajes de resistencia alcanzados por las muestras se les realiza: Graficas que describen su desarrollo a través del tiempo, diagrama de cajas para apreciar la variación entre muestras del mismo tipo y prueba Duncan para determinar semejanza entre cilindros.

\section{Resultados y discusión}

\subsection{Viabilidad del uso de agua residual tratada para mezclas de hormigón}

Todos los contaminantes analizados presentan concentraciones bajas, que no superan el máximo valor permisible de las normas ASTM C-94 y NTC 3459, con excepción de la Materia Orgánica, el contenido de Materia Orgánica medida como DQO y $\mathrm{DBO}_{5}$ superan en 11 y 6 veces respectivamente el valor máximo permisible de las Normas.

\subsection{Detección azucares, algas y sulfitos}

\subsubsection{Detección de Azúcares mediante la prueba de Molisch}

En la muestra patrón se apreció la coloración característica en la interface (coloración morada), indicando la presencia de azucares, en las muestras subsiguientes no hay coloración, poniendo en evidencia la ausencia de azucares. Concluyendo que el valor de concentración de azúcares es cero, cumpliendo con lo estipulado en las normas.

Este resultado se debe a que los microorganismos presentes, por lo general bacterias, en aguas residuales consumen rápidamente los azúcares en su metabolis- mo, las cuales son fácilmente degradadas, comparados con proteínas, grasas y aceites (Ingeniería de aguas residuales, 2000).

\subsubsection{Yodimetría para detección de Sulfitos}

La muestra de agua residual tratada, presento viraje inmediatamente después de caer una gota de titulante (equivalente a $0.001 \mathrm{~mL}$ ), esto indica una concentración mínima de sulfitos, asumida como menor a $0,001 \mathrm{mg} / \mathrm{L}$

Como el agua residual tratada es bombeada y conducida turbulentamente antes y después de la PTAR, esto ayuda a la oxigenación y como consecuencia los sulfitos al entrar en contacto con el oxígeno se oxidan a sulfatos como lo indica la prueba.

Los estados de oxidación inorgánicos más comunes en los que se encuentra el azufre en el medio ambiente varían desde el estado más reducido, -2 , característico de todos los sulfuros ( $\mathrm{S}^{2-}$, incluido naturalmente el $\left.\mathrm{H}_{2} \mathrm{~S}\right)$, hasta el más oxidado, +6 , característico de los sulfatos $\left(\mathrm{SO}_{4}{ }^{2-}\right.$, entre los que se encuentra por supuesto el $\left.\mathrm{H}_{2} \mathrm{SO}_{4}\right)($ Salas, 2005).

\subsubsection{Detección de Pigmentos- Algas}

En la placa derecha la muestra de tradescantia con líneas color rojo característico de la presencia de algas, en la placa izquierda la muestra de agua residual tratada con pocos pigmentos rojizos indica que hay presencia de estas pero en una mínima concentración. Por tal razón la concentración de algas se asume como despreciable (Figura 2).

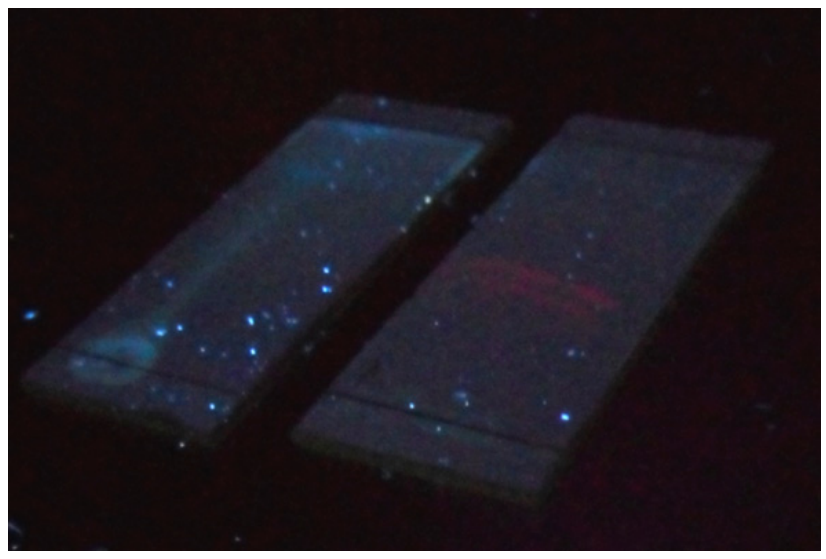

Figura 2. Placas para la detección de pigmentos. 
En el proceso de hidratación del cemento contenido en el hormigón puede alcanzar rápidamente temperaturas hasta $\operatorname{los} 30^{\circ} \mathrm{Cy} \mathrm{pH}$ superiores a 12 unidades (Moragues, 1995), es por esto, que los microorganismos y particularmente algas contenidas en el agua de mezcla en su mayoría mueren en el transcurso de esta fase.

\subsection{Análisis de resultados de laboratorio}

Para el caso del agua residual tratada dejada 8 días en reposo, la materia orgánica expresada como DQO presenta una disminución en la concentración, esto se atribuye a la degradación microbiológica de la materia orgánica.

Respecto a carbonatos de sodio y potasio, en cuerpos de agua el $\mathrm{pH}$ es un indicador de la presencia de formas predominantes de los carbonatos y bicarbonatos, cuando el pH está por debajo de 8,3 Unidades, predominan las concentraciones de bicarbonatos y con $\mathrm{pH}$ $>8,3$ Unidades empiezan a verse concentraciones representativas de carbonatos.

El valor de $\mathrm{pH}$ de la muestras están en 7,31 para PTAR y 6,8 para PTAP lo que indica mayor presencia de bicarbonatos y en menor cantidad los carbonatos. Por lo tanto la concentración de carbonatos tendrá un valor mínimo que a su vez será menor al máximo permisible de la norma. Sin embargo el valor exacto de la concentración se desconoce.

Lo mismo sucede con el agua de reposo, aunque se presenta una ligera elevación del $\mathrm{pH}$ de 7,57 Unidades, por lo anterior el valor de $\mathrm{pH}$ se encuentra en el rango establecido por las normas. Finalmente las concentraciones de estos contaminantes se presentan como no definido.

Respecto a sustancias básicas tales como el hidróxido de sodio, o sales como el potasio y el sodio (las cuales repercutirían en la resistencia del cemento); sustancias ácidas como la presencia de ácidos (clorhídrico, entre los más comunes), no debe existir preocupación ya que un $\mathrm{pH}$ entre 7,2 y 8,0 indica una sustancia neutra, esto es, que no es ni ácida ni básica, por lo tanto la concentración para estas sustancias es asumida como cero.

Con la información entregada de parámetros por parte de la PTAP El Tablazo, se aprecia que todas las concentraciones de los sustancias cumplen con los requisitos de las normas.

\subsection{Cantidad de materiales empleados en las mezclas. Tabla 1.}

Tabla 1. Materiales.

\begin{tabular}{c|c}
\hline Componentes & Peso (g) \\
\hline Grava & 30560 \\
\hline Arena & 50380 \\
\hline Cemento & 12400 \\
\hline Agua & 8270 \\
\hline Aditivo (TM-20) & 49,6 \\
\hline
\end{tabular}

\subsection{Cantidad de materiales para diseño de agua en reposo. Tabla 2.}

Tabla 2. Materiales.

\begin{tabular}{c|c}
\hline Componentes & Peso (g) \\
\hline Grava & 31130 \\
\hline Arena & 53140 \\
\hline Cemento & 12400 \\
\hline Agua & 5000 \\
\hline Aditivo (TM-20) & 49,6 \\
\hline
\end{tabular}

La variación en cantidades de materiales corresponde al hecho de que la humedad de los agregados varia, ya que estas mezclas fueron elaboradas en días distintos.

\subsection{Resultados de pruebas a la compresión}

En el anexo 1 se encuentran los valores de la fuerza ejercida, el área de presión de los cilindros y los porcentajes de presión alcanzados respecto a cilindros con agua potable, según el tipo de mezcla, para las pruebas realizadas a $\operatorname{los} 7,14$ y 28 días.

\subsubsection{Variación de la resistencia respecto al tiem- po para muestras DAP, DAR y D50/50. Figura 3.}

A los 7 días se observa que la resistencia de todos los diseños difiere levemente entre sí. Para el día 14 las muestras DAP y D50/50 presentan semejanza, caso contrario para DAR que empieza a distanciarse levemente de los valores de resistencia alcanzados por DAP. Finalmente a los 28 días se observa que D50/50 presenta un leve aumento de resistencia respecto a DAP. 


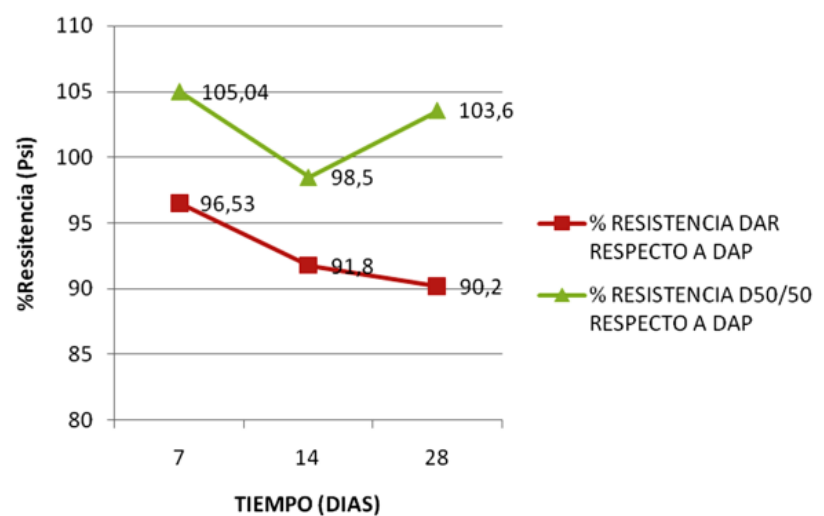

Figura 3. Variación de la resistencia.

La resistencia de DAR es menor respecto a las resistencias alcanzadas por DAP y D50/50, esto se aprecia tanto a $\operatorname{los} 7,14$ y 28 días. DAP y D50/50 tienen resistencias similares en todos los periodos de tiempo. Al parecer después de 21 días la resistencia de D50/50 podría aumentar y superar la resistencia de DAP.

\subsubsection{Dispersión de la resistencia demuestras DAP, DAR y D50/50.}

$\operatorname{DAR}(1,2,3)$ diseño a $\operatorname{los} 7,14$ y 28 días respectivamente.

$\mathrm{D} 50 / 50(1,2,3)$ diseño a $\operatorname{los} 7,14$ y 28 días respectivamente.

DAP $(1,2,3)$ diseño a los 7, 14 y 28 días respectivamente. Figura 4.

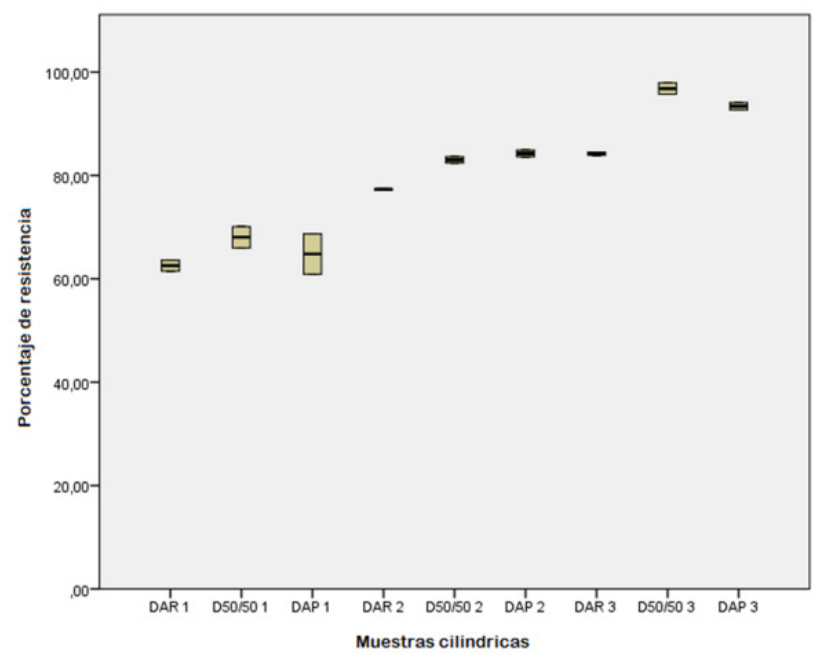

Figura 4. Dispersión en las resistencias
Se puede observar mediante el diagrama de cajas que existe mayor dispersión en muestras fracturadas a los 7 días en la prueba de resistencia, esta dispersión es mayor en la muestra DAP. Para las muestras fracturadas a los 14 días se aprecia mínima dispersión entre las muestras el mejor representante de esto es DAR.

A los 28 días las muestras presentan poca dispersión, aunque se puede observar que los valores con resistencias más dispersas los obtuvieron las muestras de D50/50. Estas variaciones pueden deberse a una mala manipulación de la máquina de pruebas.

\subsubsection{Similitud entre muestras DAP, DAR y D50/ 50. Tabla 3 .}

El primer grupo obtenido entre DAR1 y DAP1 son semejantes en resistencia, el grupo 2 obtenido entre DAP 1 y D50/50 1 son semejantes en resistencia y a la vez son distintos del grupo 1 y de los demás grupos generados.

En el grupo 4 se aprecia que las resistencias de D50/ 502 y DAP 2 son semejantes y simultáneamente a un DAR 3. Significa que la resistencia de DAR 3 perteneciente al periodo de 28 días tuvo un retardo para alcanzar la resistencia de sus similares de 14 días. Por último DAP y D50/50 son semejantes a los 28 días.

Los retardos y reducción de resistencia de las muestras DAR son debidas seguramente a las altas concentraciones de materia orgánica presentes en el agua de mezcla.

3.6.4 Variación de la resistencia respecto al tiempo para muestras DAP, DARR y D50/50R (agua en reposo) (Figura 5).

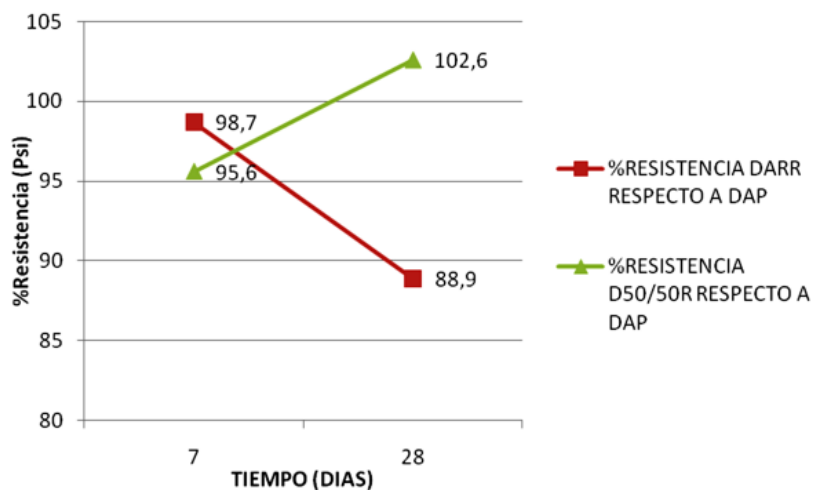

Figura 5. Variación de la resistencia. 
Tabla 3. Similitud entre muestras

Resistencia

\begin{tabular}{|c|c|c|c|c|c|c|}
\hline \multirow[b]{2}{*}{ Cilindro2 } & \multirow[b]{2}{*}{$N$} & \multicolumn{5}{|c|}{ Subconjunto para alfa $=0.05$} \\
\hline & & 1 & 2 & 3 & 4 & 5 \\
\hline DAR 1 & 2 & 62,5500 & & & & \\
\hline DAP 1 & 2 & 64,8000 & 64,8000 & & & \\
\hline $\mathrm{D} 50 / 501$ & 2 & & 68,0500 & & & \\
\hline DAR 2 & 2 & & & 77,3000 & & \\
\hline $\mathrm{D} 50 / 502$ & 2 & & & & 83,0000 & \\
\hline DAR 3 & 2 & & & & 84,2000 & \\
\hline DAP 2 & 2 & & & & 84,2500 & \\
\hline DAP 3 & 2 & & & & & 93,4000 \\
\hline $\mathrm{D} 50 / 503$ & 2 & & & & & 96,8000 \\
\hline Sig. & & ,347 & 185 & 1,000 &, 610 & 168 \\
\hline
\end{tabular}

Se visualizan las medias para los grupos en los subconjuntos homogéneos.

a. Utiliza el tamaño de la muestra de la media armónica $=2,000$.

Todos los diseños demuestran que tienen valores de resistencia semejantes a los 7 días, aunque con leve reducción en D50/50R. A los 14 días se nota como la resistencia para $\mathrm{D} 50 / 50$ es similar a la resistencia para DAP, mientras que la resistencia de DARR es inferior respecto a los demás diseños.

El día 28 se observa como D50/50R es similar en su resistencia a DAP, mientras que DARR queda varias unidades por debajo de estas. El desarrollo en resistencia de D50/50R es similar a DAP hasta los 21 días, después de este tiempo se aprecia un leve incremento en la resistencia.

\subsubsection{Dispersión de la resistencia de muestras} $D A P, D A R R$ y D50/50R (agua en reposo). Figura 6.

Las muestras DAP 4 y D50/50R 5 presentan mayor dispersión respecto a las demás muestras cilíndricas, además de esto los rangos de dispersión entre estas dos muestras son similares.

\subsubsection{Similitud entre muestras DAP, DARR y D50/ 50R. Tabla 4.}

El primer grupo está compuesto por todos los diseños a los 7 días, es decir todos los diseños son semejantes a los 7 días. DARR 5 es totalmente diferente a todos

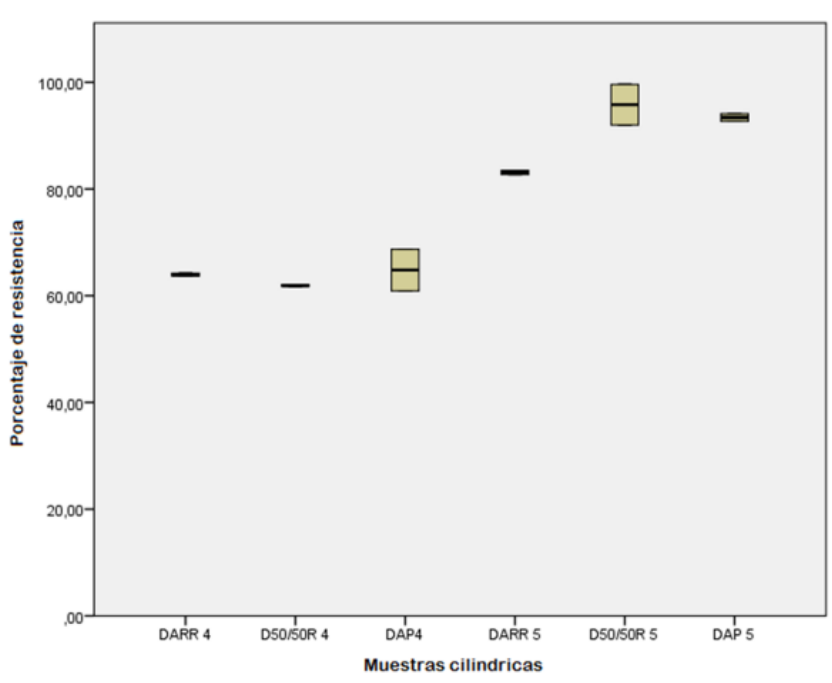

Figura 6. Dispersión de la resistencia.

los demás diseños a los 28 días. DAP 5 y D50/50R 5 son semejantes a los 28 días. De esta manera se puede definir que DAP y D50/50R tienen resistencias similares en todos los periodos de tiempo analizados.

\subsubsection{Variación de la resistencia respecto al tiempo para muestras DAP, DAR, D50/50, DARR y D50/50R}

A continuación se observa en la figura 7 el comportamiento de la resistencia alcanzada de todas las mez- 
Tabla 4. Similitud entre muestras.

Resistencia

Duncan $^{\mathrm{a}}$

\begin{tabular}{l|r|c|c|c}
\hline & \multirow{2}{*}{ Cilindro2 } & N & \multicolumn{3}{|c}{ Subconjunto para alfa $=0.05$} \\
\cline { 3 - 5 } & 2 & 61,9000 & & 3 \\
\hline D50/50R 4 & 2 & 63,9500 & & \\
DARR 4 & 2 & 64,8000 & & \\
DAP4 & 2 & & 83,1000 & \\
DARR 5 & 2 & & & 93,4000 \\
DAP 5 & 2 & & & 95,8000 \\
D50/50R 5 & & & &, 479 \\
Sig. & &, 411 & 1,000 & \\
\hline
\end{tabular}

Se visualizan las medias para los grupos en los subconjuntos homogéneos.

a. Utiliza el tamaño de la muestra de la media armónica $=$ 2,000 .

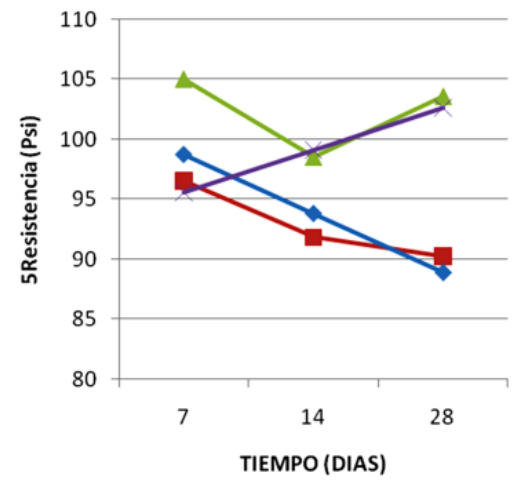

$\%$ RESISTENCIA DAR RESPECTO A DAP

- $\%$ RESISTENCIA D50/50 RESPECTO A DAP

$\sim$ \%RESISTENCIA DARR RESPECTO A DAP

-\%RESISTENCIA D50/50R RESPECTO A DAP

Figura 7. Variación de la resistencia.

clas de hormigón elaboradas, respecto al periodo de tiempo de 7 a 28 días. Finalmente con este análisis se determina las similitudes entre las resistencias alcanzadas por las muestras elaboradas con agua residual tratada reposada y no reposada.

Luego de 7 días DAR y DARR tienen desarrollo similar hasta los 28 días pero con los menores valores en resistencia presentados respecto a DAP.

D50/50 y D50/50R presentaron diferencias entre sus resistencias desde los 7 días hasta poco antes de los 28 días, presentando igual valor de resistencia al día 28; a pesar de sus diferencias demostraron tener resistencia similar o superior a DAP. Se evidencia que el agua reposada durante 8 días no afecta significativamente en la resistencia del hormigón.

\subsubsection{Dispersión de la resistencia de muestras DAR, D50/50, DAP, DARR y D50/50R (Figura 8).}

La mayor dispersión se presentó en los diseños, D50/ 50 1, DAP 1 y D50/50R 2, los demás diseños presentan menor dispersión respecto a estos.

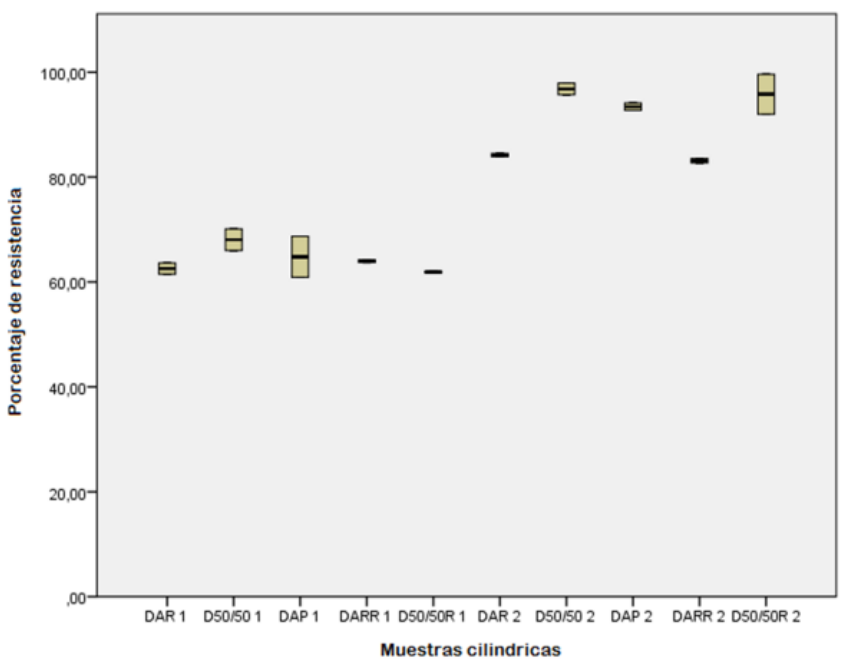

Figura 8. Dispersión de la resistencia.

\subsubsection{Similitud entre muestras DAR, D50/50, DAP, DARR y D50/50R. Tabla 5.}

El primer grupo indica semejanza de resistencia de todos los diseños a los 7 días; el segundo grupo está

Tabla 5. Similitud entre resistencias.

Resistencia

Duncan $^{a}$

\begin{tabular}{l|r|c|c|c}
\hline & \multirow{2}{*}{ Cilindro2 } & $\mathrm{N}$ & \multicolumn{3}{|c}{ Subconjunto para alfa $=0.05$} \\
\cline { 3 - 5 } D50/50R 1 & 2 & 61,9000 & 2 & 3 \\
DAR 1 & 2 & 62,5500 & & \\
DARR 1 & 2 & 63,9500 & & \\
DAP 1 & 2 & 64,8000 & & \\
D50/50 1 & 2 & 68,0500 & & \\
DARR 2 & 2 & & 83,1000 & \\
DAR 2 & 2 & & 84,2000 & \\
DAP 2 & 2 & & & 93,4000 \\
D50/50R 2 & 2 & & & 95,8000 \\
D50/50 2 & 2 & & & 96,8000 \\
Sig. & & & & \\
\hline
\end{tabular}

Se visualizan las medias para los grupos en los subconjuntos homogéneos.

a. Utiliza el tamaño de la muestra de la media armónica = 2,000 . 
constituido por los DAR y DARR de 28 días, indicando que estos son semejantes a pesar de que son realizados con agua de reposo y sin reposar.

Los valores de resistencia de D50/50 y D50/50R de 28 días son semejantes. Esto reafirma el hecho de que el agua residual tratada de reposo no afecta significativamente en la resistencia respecto una no reposada, simultáneamente estos diseños son semejantes en resistencia a DAP.

DAR y DARR presentan la menor resistencia obtenida y está por debajo de DAP, esto hace que el uso en una proporción total de agua residual tratada en mezclas de hormigón sea una alternativa posible pero de cuidado y mayor investigación, ya que se encuentra en el límite del valor aceptado por norma INVIAS 630 de 2013.

Cuando se diluye una cantidad de agua residual tratada a la mitad, también se diluyen los contaminantes que esta contiene, en este caso el contaminante que influyó directamente en las mezclases la materia orgánica, ya que su concentración está muy por encima de lo estipulado en las normas.

Con el uso de agua residual tratada en todos los diseños se observó un retraso en el fraguado, esto no necesariamente es algo negativo; las empresas de premezclados usan aditivos que retardan el fraguado o endurecimiento debido a que transportan sus mezclas a lugares que pueden ser lejanos, por tal razón si no controlan el fraguado de la mezcla puede llegar endurecida a su punto de destino, lo cual afectaría drásticamente una futura estructura. Por lo tanto se puede definir que el uso de agua residual tratada en un $50 \%$ tiene un efecto como aditivo natural.

La forma de rompimiento o falla de las muestras cilíndricas son de tipo falla lateral en su mayoría y de tipo agrietamiento de columnado en menor cantidad.

\section{Conclusiones}

Las características físicoquímicas del agua residual tratada de la PTAR Cañaveralejo, durante el año 2015 e inicios del 2016, a pesar de presentar variaciones estacionales, mantienen un comportamiento regular y las concentraciones son aceptables para la preparación de mezclas de hormigón según las Normas ASTM C-94 y NTC 3459, excepto el contenido de materia orgánica medido como $\mathrm{DQO}$ y $\mathrm{DBO}_{5}$ que superan en
11 y 6 veces respectivamente el valor máximo permisible de las Normas. El agua potable suministrada por la PTAP El Tablazo presenta sustancias con concentraciones que no superan los valores máximos permisibles de las Normas ASTM C-94 y NTC 3459, haciéndola ideal para mezclas de hormigón. Las muestras preparadas con partes iguales de Agua Residual Tratada y Agua Potable, en reposo como sin reposar, presentaron resistencia a los 28 días similar a la alcanzada por los cilindros fabricados con Agua Potable. Esto indica que es viable emplear este tipo de agua residual con estas características, para preparar mezclas de hormigón. Las resistencias obtenidas a los 28 días de los cilindros preparados con el $100 \%$ agua residual tratada y agua residual tratada en reposo fueron del $90,2 \%$ y $88,9 \%$ respectivamente, respecto a la resistencia obtenida con el agua potable; valores que se encuentran por debajo de lo estipulado en las normas $(>92 \%)$. Por lo tanto no es apropiado usarla directamente en mezclas de hormigón. Si la PTAR Cañaveralejo realizara un proceso adicional de tratamiento que redujera la concentración de materia orgánica expresadas en $\mathrm{DBO}_{5}$ y DQO en forma significativa ( $>50 \%$ ), posiblemente la resistencia del hormigón con uso total de agua residual tratada mejoraría. No hay diferencias significativas en la resistencia a los 28 días entre el hormigón realizado con agua residual en reposo de 8 días y sin reposar, con lo cual se evidencia que no hay alteración significativa en sus características fisicoquímicas durante este tiempo.

\section{Referencias bibliográficas}

Acevedo, H., Vásquez, A., Ramírez, D. 2012. Sostenibilidad. Actualidad y necesidad en el sector de la construcción en Colombia. p: 2. Tomado de internet: http:/ /www.revistas.unal.edu.co/index.php/gestion/article/ view/30825/39307

Al-Ghusain, I., Terro, M., 2003. Use of treated wastewater for concrete mixing in Kuwait. Civil Engineering Department, Kuwait University. p 9-11. Tomado de internet: http://www.sciencedirect.com/ science/article/pii/S0956053X04001126

American Society for Testing and Materials, ASTM C94.C-94/M 04.Standard Specification for ReadyMixed Concrete, p. 14.

Cámara Colombiana de la construcción CAMACOL. 2014. Informe económico una publicación mensual ¿qué nos indican las brechas del mercado edificador 
para el 2014? cierre 2013 y perspectivas 2014 No 56. p 1. Tomado de internet: http://camacol.co/sites/ default/files/secciones internas/Informe $\% 20$ Econ $\% \mathrm{C} 3 \% \mathrm{~B} 3 \mathrm{mico} \% 20 \mathrm{Marzo} \% 202014 \% 20-\% 20 \mathrm{No} \%$ $20 \% 2056 . p d f$

Diéguez, V., 2011. Propiedades físicas del concreto elaborado con agua residual tratada. Universidad central de Venezuela, Caracas Venezuela. p. 20.

Gadzama, E., Ekele, O., Anametemfiok, V., \& Abubakar, A. (2015) Effects of sugar factory wastewater as mixing water on the properties of normal strength concrete. Civil Engineering Department. Modibbo Adama University of Technology, P.M.B. 2076 Yola, Adamawa State, Nigeria. p 2,3. Tomado de internet: http://www.iosrjournals.org/iosrjmce/papers/vol13issue6/Version-2/G1306024145.pdf

Ingeniería de aguas residuales., 2000, p. 51. Consultado en. http://laoropendolasostenible.blogspot.com.co/ 2012/12/ingenieria-de-aguas-residuales-pdf.html

Instituto nacional de vías. INVIAS. Especificaciones generales de construcción de carreteras y normas de ensayo para materiales de carreteras. Tomado de internet: http://www.invias.gov.co/index.php/documentos-tecnicos-izq

Medina, C., 2013. Concreto confeccionado con aguas lluvia: Un aporte a la disminución del impacto ambiental generado por la industria de la construcción. Universidad nacional de Colombia. Medellín.2013.p 8. Consultado en. http://www.bdigital.unal.edu.co/ 9593

Moragues, A., 1995. Procesos de hidratación del cemento Portland y microestructura del hormigón. Universidad politécnica de Madrid. Tomado de internet: http://prt2.uprm.edu/Microestructura.pdf

Naik, T., 2007. Sustainability of the cement and concrete industries: Sustainable construction materials and Technologies. p 19.Consultado en. https:// www4.uwm.edu/cbu/Papers/2004\%20CBU\%20 Reports/CBU-2004-15.pdf
Norma Técnica Colombiana., N.T.C. 30., 2001 Cemento Pórtland. Clasificación y Nomenclatura. Tomado de internet: http://www.academia.edu/9500250/ NTC-30-Cemento-Portland-Clasificacion-yNomenclatura.

Norma Técnica Colombiana., N.T.C. 3459. 2001. Aguas para la elaboración de concreto.

Oviedo, L., 2011. Estudio de alternativas de reúso y reciclo de aguas residuales en aplicaciones industriales y municipales. Universidad industrial de Santander. Bucaramanga. p 15. Tomado de internet: http:// repositorio.uis.edu.co/jspui/bitstream/123456789/ 7113/2/142273.pdf

Polanco, J., Setién, J., 2012. Cementos, morteros y hormigones. Ciencia e ingeniería del terreno y de los materiales. Tomado de internet: http://ocw.unican.es/ ensenanzas-tecnicas/cementos-morteros-y-hormigones/materiales/3_Morteros_hormigones1.pdf

Rivera, G., 2000. Concreto simple. Universidad del Cauca. Tomado de internet: https://inforcivilonline. wordpress.com/2015/05/23/concreto-simple-inggerardo-a-rivera-1/D

Salas G., 2005. Eliminación de sulfuros por oxidación en el tratamiento del agua residual de una curtiembre. Tomado de internet: http://sisbib.unmsm.edu.pe/ bibvirtual/publicaciones/ing_quimica/v08_n1/pdf/ a08v8.pdf

Standard Methods for the Examination of Water and Wastewater, Edición 22.

Vásquez, A., Gonzales, F., Rocha, L. y Flores, A. 2001. Elaboración de concretos con aguas tratadas. México.

Zika. 2012. HOJA TECNICA Versión: 01. Plastiment TM 20 , Retardador plastificante-Reductor de agua. 
ANEXO 1. RESULTADOS PRUEBAS DE COMPRESION

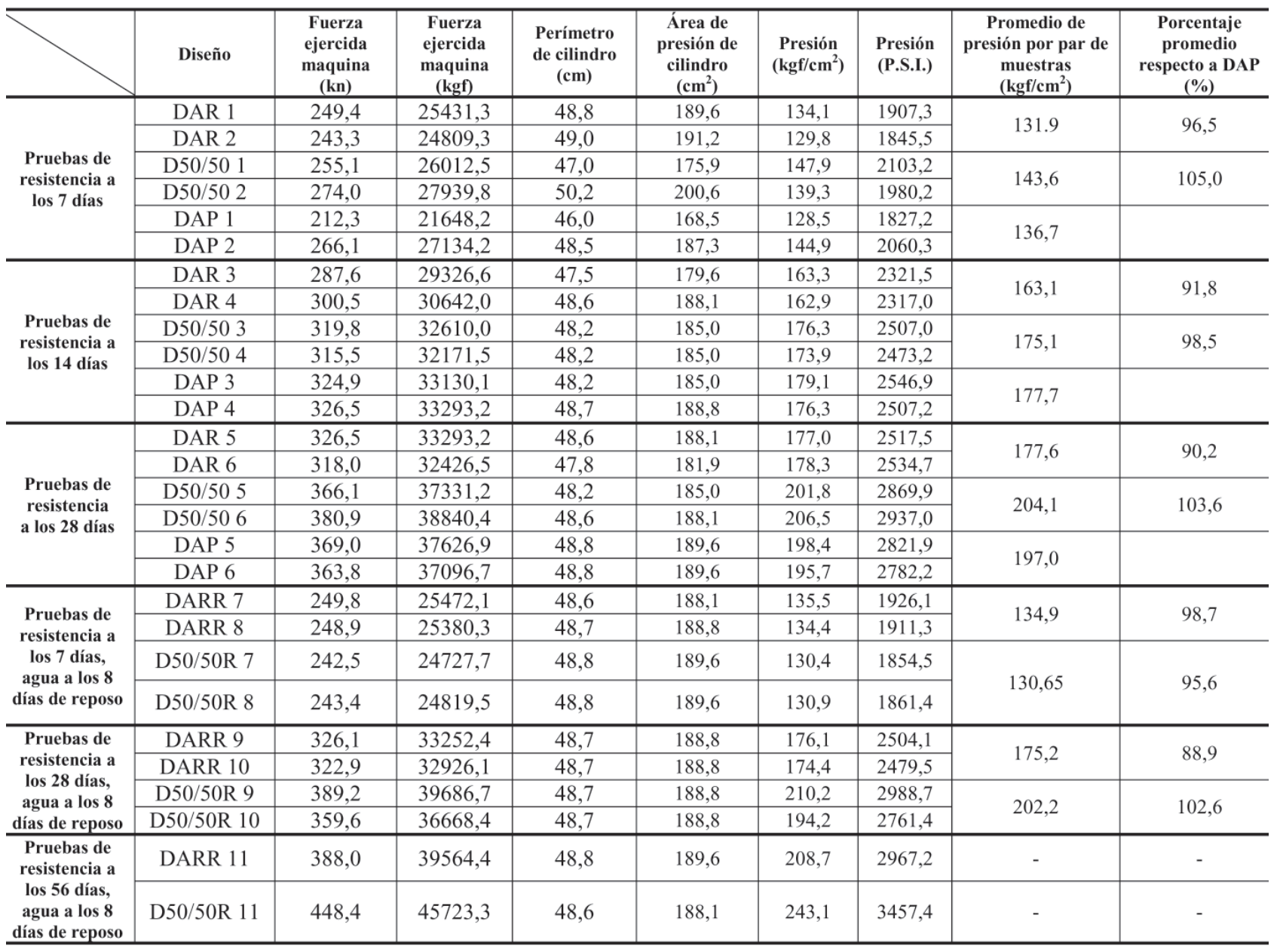

DAR: Diseño con agua residual tratada

D50/50: Diseño con $50 \%$ de agua residual tratada y $50 \%$ de agua potable

DAP: Diseño con agua potable

DARR: Diseño con agua residual tratada de reposo

D50/50R: Diseño con 50\% de agua residual tratada de reposo y $50 \%$ de agua potable 